\title{
Observation of the propagation and interference of spin waves in ferromagnetic thin films
}

\author{
Korbinian Perzlmaier, Georg Woltersdorf, ${ }^{*}$ and Christian H. Back \\ Institut für Experimentelle und Angewandte Physik, Universität Regensburg, 93040 Regensburg, Germany
}

(Received 7 December 2007; published 20 February 2008)

\begin{abstract}
We have observed the propagation of spin waves and spin-wave packets in 20-nm-thick Permalloy films upon microwave excitation by means of time-resolved scanning Kerr microscopy. Spin waves can be detected up to $80 \mu \mathrm{m}$ away from the driving coplanar strip line. In doing so, we were able to directly determine the phase velocities, dispersion relations and group velocities for different geometries of an externally applied in-plane magnetic field. In addition, we report on the direct optical observation of spin-wave interference with lateral and time (phase) resolution.
\end{abstract}

DOI: 10.1103/PhysRevB.77.054425

PACS number(s): 75.30.Ds, 75.50.Bb, 75.70.Ak, 76.50.+g

The elementary excitations in thin ferromagnetic films are spin waves which possess peculiar dispersion relations. In particular, at low $\vec{k}$ vectors in the plane of a thin film and with the magnetization also in plane, the spin-wave dispersion relations show positive and negative dispersion resulting in positive and negative group velocities. In magnetic systems, negative group velocity was predicted theoretically ${ }^{1-3}$ and excitations with frequencies lower than the uniform precessional mode were interpreted as magnetostatic backward modes. ${ }^{4-9}$ In ferromagnetic systems it may be possible to form a junction between a magnetic entity having positive to a region having negative dispersion. In fact, in magnetic systems one can easily jump from one branch of the dispersion relation to another by changing the direction of the equilibrium magnetization while keeping the $\vec{k}$ vector of the propagating spin waves constant. Stable states for the equilibrium magnetization can be designed by employing e.g. shape or magnetocrystalline anisotropy. As magnetic switching between two stable states can be performed at subnanosecond time scales, ${ }^{10,11}$ high-speed elementary spin logic such as spin-wave switches, isolators, circulators, resonators, filters or phase shifters may be realized.

In recent years, spin-wave propagation and the propagation of nonlinear spin-wave packets (bullets or spin-wave solitons) have been investigated thoroughly for ferrimagnetic insulators such as yttrium iron garnet (YIG). ${ }^{12}$ In this insulating material the intrinsic damping can be very small ( $\alpha$ $=0.0003$ ) allowing the transport of magnetic information over large distances, in some cases millimeters. Unfortunately, for miniaturization and easy integration into electronic circuits, this class of materials does not appear to be adequate, as high-quality YIG crystals are usually grown from the liquid phase and cannot be sputtered or evaporated. On the other hand, a material that possesses a fairly small intrinsic damping $(\alpha=0.008)$ is Permalloy $\left(\mathrm{Ni}_{80} \mathrm{Fe}_{20}\right)$. This material-being a standard metal-may easily be used in standard lithography processes. In fact, recent publications employing inductive detection demonstrate the propagation of spin waves in ferromagnetic thin $\mathrm{Ni}_{80} \mathrm{Fe}_{20}$ films ${ }^{13,15,14,16}$ over rather large distances of up to $50 \mu \mathrm{m}$ or-by secondharmonic magneto-optical methods-even over distances of hundreds of micrometers. ${ }^{17}$ Using time-resolved (TRMOKE) magneto-optical Kerr effect scanning microscopy the propagation of spin waves has recently been observed directly in thin $\mathrm{Ni}_{80} \mathrm{Fe}_{20}$ films for two distinct geometries. ${ }^{18}$

In this paper we report on the propagation of spin waves in 20-nm-thick continuous $\mathrm{Ni}_{80} \mathrm{Fe}_{20}$ films. By means of TRMOKE microscopy, we detect the propagation of spin waves excited by continuous-wave (cw) or pulsedmicrowave fields up to $80 \mu \mathrm{m}$ away from a coplanar strip (CPS) line. From the acquired data, we were able to extract the phase velocities, dispersion relations and group velocities of spin-wave pulses. The experimentally obtained results can be reproduced quantitatively by analytical calculations. In addition, we demonstrate the direct optical observation of the interference of spin waves in ferromagnetic thin films with lateral and temporal (relating to the phase of the oscillation) resolution.

The sample considered in the following consists of a 20nm-thick $\mathrm{Ni}_{80} \mathrm{Fe}_{20}$ film with lateral dimensions of 200 $\times 200 \mu \mathrm{m}^{2}$ patterned onto a GaAs substrate using electron beam lithography, thermal evaporation and subsequent liftoff. In a second step, a 200-nm-thick Au CPS is patterned over one edge of the $\mathrm{Ni}_{80} \mathrm{Fe}_{20}$ film using optical lithography and lift-off [see Fig. 1(a)].

We use the magneto-optic Kerr effect to detect the magnetic excitation of the sample. In doing so, we use a modified TRMOKE setup similar to the one described in a previous paper. ${ }^{19,20}$ The TRMOKE setup with a spatial resolution of $300 \mathrm{~nm}$ and a temporal resolution better than $1 \mathrm{ps}$ has been modified such that a microwave generator is synchronized to
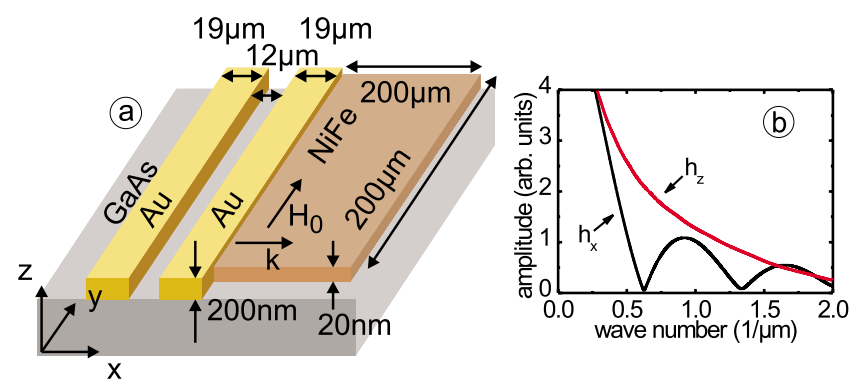

FIG. 1. (Color online) (a) Sample layout. The CPS is placed over the edge of the $\mathrm{Ni}_{80} \mathrm{Fe}_{20}$ film. (b) Wave-number distribution of the $x$ and $z$ components of the magnetic microwave field created by the CPS. The wave-number distribution is calculated numerically and is very sensitive to the overlap between the magnetic film and CPS. This overlap is determined by optical microscopy. 
the $80-\mathrm{MHz}$ repetition rate of the Ti:sapphire laser system, which enables us to establish a phase-stable correlation between microwave (MW) excitation and laser probe pulse. Our frequency resolution is thus limited to $80 \mathrm{MHz}$. The amplified sinusoidal signal of the microwave generator is used to excite the magnetization by the magnetic field generated by the rf current in the CPS. For some experiments a frequency mixer is added into the microwave excitation line which allows us to excite the magnetization with microwave field pulses (MWPs) as short as $1 \mathrm{~ns}$ at a carrier frequency given by a multiple of the $80-\mathrm{MHz}$ repetition rate. It is important to note that we use MWP excitation with a phasestable starting condition with respect to the laser repetition rate. The microwave packets are subsequently amplified the same way as the cw excitation.

The excitation of the magnetic film is detected as function of various parameters (time, position, bias field, and excitation frequency). Resonance scans are scans in time at one specific position of the film. By measuring the amplitude of the $z$ component of the precessional motion of the magnetization in response to the MW excitation as a function of MW frequency at a fixed MW power, resonance curves can be recorded and the resonance frequency of the magnetic system can be determined. Line scans are scans in time and position. It is thus possible to observe the propagation of a spin-wave packet directly. Finally images as a function of time can be recorded allowing one to observe the propagation of spin-wave packets in space and time.

The response of a magnetic structure to an applied field is given by the magnetic susceptibility. In the case of cw excitation, the high-frequency (hf) susceptibility needs to be considered which is given by $\chi_{i j, G}=\frac{m_{i, G}}{h_{j, G}}$. Here, $m_{i, G}$ denotes the component of the dynamic magnetization pointing along the $i$ direction and $h_{j, G}$ is the hf magnetic field component along the $j$ direction. The subscript $G$ represents the particular geometry of the magnetic excitation. Note that the explicit forms of $m_{i, G}$ and $h_{j, G}$ depend on the experimental geometry. In particular two cases are usually considered in the thin film limit: (i) the Damon-Eshbach (DE) geometry where the direction of the static magnetization $\vec{M}$ and the propagation direction of the spin waves $\vec{k}$ are perpendicular to each other, but in the film plane, and (ii) the magnetostatic-backwardvolume (MSBV) geometry where the two vectors are collinear within the film plane.

The susceptibilities can be calculated from the effective magnetic fields-i.e. the external bias field $H_{0}$, the exchange field, and the demagnetizing field (which is calculated following the approach by $\mathrm{Harte}^{21}$ ) - and the linearized Landau-Lifshitz-Gilbert (LLG) equation.

$$
\chi_{z x}^{\mathrm{DE}}=\frac{-i \frac{\omega}{\mu_{0} \gamma} M_{s}}{\left(\frac{2 A k^{2}}{\mu_{0} M_{s}}+M_{s} \frac{1-e^{-k d}}{k d}+H_{0}-i \alpha \frac{\omega}{\mu_{0} \gamma}\right)\left(\frac{2 A k^{2}}{\mu_{0} M_{s}}+M_{s} \frac{k d-1+e^{-k d}}{k d}+H_{0}-i \alpha \frac{\omega}{\mu_{0} \gamma}\right)-\frac{\omega^{2}}{\left(\mu_{0} \gamma\right)^{2}}} e^{-i(k x-\pi / 2)}
$$

and

$$
\chi_{z z}^{\mathrm{MSBV}}=\frac{M_{s}\left(\frac{2 A k^{2}}{\mu_{0} M_{s}}+H_{0}-i \alpha \frac{\omega}{\mu_{0} \gamma}\right)}{\left(\frac{2 A k^{2}}{\mu_{0} M_{s}}+M_{s} \frac{1-e^{-k d}}{k d}+H_{0}-i \alpha \frac{\omega}{\mu_{0} \gamma}\right)\left(\frac{2 A k^{2}}{\mu_{0} M_{s}}+H_{0}-i \alpha \frac{\omega}{\mu_{0} \gamma}\right)-\frac{\omega^{2}}{\left(\mu_{0} \gamma\right)^{2}}} e^{-i(k x+\pi / 2)},
$$

where $A=1.05 \times 10^{-11} \mathrm{~J} / \mathrm{m}$ is the exchange constant, $\mu_{0} M_{s}=1 \mathrm{~T}$ the saturation magnetization, $H_{0}$ the external magnetic bias field, $\gamma=17.6 \mathrm{GHz} / \mathrm{T}$ the gyromagnetic ratio, $d$ the thickness of the film, and $\alpha$ the damping parameter of $\mathrm{Ni}_{80} \mathrm{Fe}_{20}$. Setting the denominators to zero and solving the resulting equations for $\omega=2 \pi f$ and $k$, one obtains the response which is referred to as the "analytic" dispersion relation in the course of this paper. Combining the above susceptibilities with the local excitation spectrum of the CPS which is calculated numerically, the "expected response" of the magnetic film can be calculated and compared to experimental data.

Thus it is obvious that the response of the magnetic system depends on the spatial Fourier components of the rf driving field generated by the CPS. In the coordinate system adopted here, the CPS creates high-frequency microwave fields with components $h_{x}$ and $h_{z}$ [see Fig. 1(b)] around its profile. Spin waves are expected to propagate from the CPS along the $x$ direction, with wave vectors $\vec{k}=(k, 0,0)$. An external static magnetic in-plane bias field $\vec{H}_{0}$ is applied to the sample along the $x$ direction for the MSBV geometry and along the $y$ direction for the DE geometry.

We can now place the probing laser spot at a position $10 \mu \mathrm{m}$ away from the CPS and record the resonance as a function of frequency in an applied external bias field of 180 Oe along the $y$ direction (DE geometry). The data are shown in Fig. 2(a), black circles and black line. A clear resonance peak can be observed at about $4 \mathrm{GHz}$. The amplitude $m_{z}^{0}\left(f_{0}\right)$ of the resulting response at a fixed frequency $f_{0}$ can be straightforwardly calculated by integrating the product of the amplitudes of the wave-number components of the 

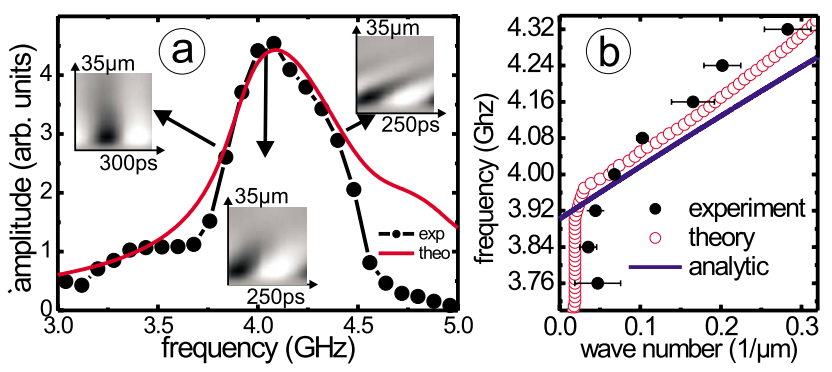

FIG. 2. (Color online) (a) Resonance of the $\mathrm{Ni}_{80} \mathrm{Fe}_{20}$ film, obtained by scanning Kerr microscopy (black line and black circles) and calculated response (red line) for a magnetic in-plane bias field of 180 Oe in the DE geometry. The insets show line scans taken at the indicated frequencies. The line scans start at the edge of the CPS and reach $35 \mu \mathrm{m}$ into the continuous film. From the slope of these scans, the dispersion relation can be determined. The discrepancy between experiment and theory for frequencies above the resonance is due to the limited bandwidth of the amplifier-waveguide system. (b) Maximum magnetic response determined from the experiments (black circles) and compared to the calculated response (red open circles). The blue line represents the analytic dispersion relation.

driving magnetic field $h(k)$ and the respective value of the susceptibility $\quad \chi\left(f_{0}, k\right) \quad$ which leads to $m_{z}^{0}\left(f_{0}\right)=\int_{k} h_{j}(k) \chi_{z j}\left(f_{0}, k\right) d k$. Note that we always measure the $z$ component of magnetization in our experimental setup.

We would like to emphasize that in our experiments we measure forced oscillations of the magnetization. Therefore, the dynamics can be excited at arbitrary frequencies (even far away from resonance). The response of the magnetization is given by the product of the $\vec{k}$-dependent excitation [see Fig. 1(b)] and the $\vec{k}$-dependent susceptibility. The $\vec{k}$ vector where this product has a maximum is determined in our experiment. In the DE geometry, for instance, with its monotonically positive dispersion, no wave number is defined by the analytic dispersion below the resonance frequency. However, in our experiments spin waves can also be driven at frequencies below resonance and a dominating wave number can be identified. Our theoretical model reproduces this behavior well (see Fig. 2).

While the frequency can be chosen freely with an increment of $80 \mathrm{MHz}$, the respective wave number of the excitation is always given by the CPS geometry. This means that the magnetic film "picks" the wave vector with the largest susceptibility from the Fourier spectrum of the CPS at a given driving frequency. In the insets of Fig. 2(a) we show line scans as a function of time. Using these data the phase velocity $v_{P h}$ of the spin waves can be directly determined from the slope $\Delta x / \Delta t$ of the decaying oscillatory signal. $v_{P h}$ decreases with increasing frequency. The phase velocity can easily be converted into a $\vec{k}$ vector: $k=\frac{2 \pi f}{v_{p h}}$. Plotting frequency versus wave number [see Fig. 2(b)] results in the dispersion relation for the observed spin waves. The data are plotted along with the dispersion relation obtained by our calculation as discussed above. While the analytic dispersion represents the line of maximum values in the susceptibility for given $\vec{k}$ vectors, the experimentally obtained dispersion deviates from these values. The reason for this is to be found

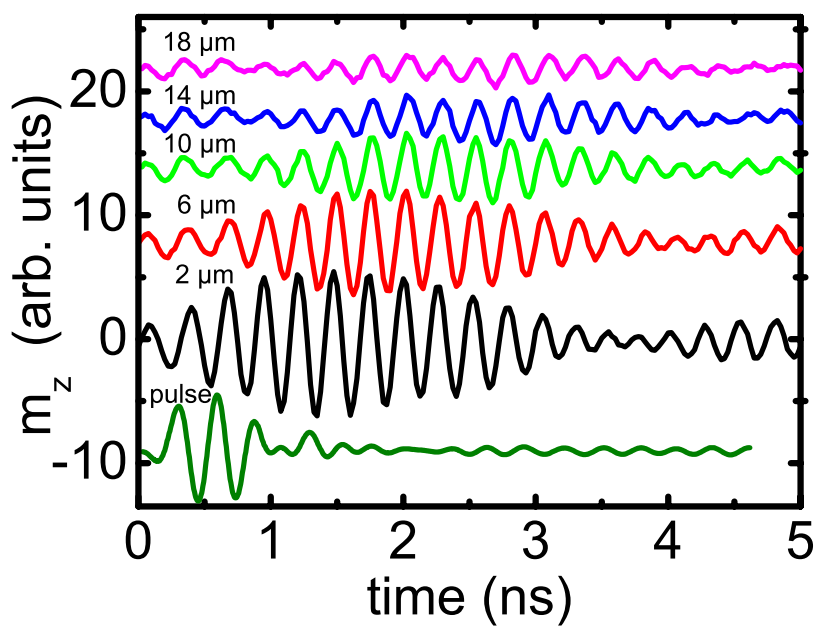

FIG. 3. (Color online) Propagation of spin-wave packets. Profile of the microwave pulse (lowest line) and scans in time of the spinwave packet at various distances from the CPS (the distance in microns is indicated above the individual lines). The curves have been offset for clarity.

in the inhomogeneous wave-number distribution of the microwave field and the possibility to excite spin waves with frequencies lower than the resonance frequency expected for $k=0$. This behavior is also well reproduced by our theoretical model [see Fig. 2(b)].

As expected for the DE geometry, the frequency increases with increasing wave number. In the MSBV geometry data, however, only very small wave numbers very close to zero are observed. The reason for this behavior is the shallow dispersion of the MSBV. Since the Fourier spectrum of the excitation mainly contains small $\vec{k}$ vectors and the dispersion is flat, it is not possible to significantly excite high- $\vec{k}$-vector spin-wave modes and the small wave numbers dominate. For the $\vec{k}$-vector range studied in this paper, the dispersion is much steeper in the DE geometry than in the MSBV geometry. Only the steeper dispersion in the DE geometry allows one to excite spin waves with lager $k$ vectors.

On the same sample one can now perform experiments with pulsed-MW excitation. Delay scans at various distances from the CPS are shown in Fig. 3. It can clearly be observed how the MW pulse triggers a spin-wave packet that propagates along the $x$ direction and broadens considerably as a function of time due to dispersion. In addition, the signal is damped according to the intrinsic damping parameter of the $\mathrm{Ni}_{80} \mathrm{Fe}_{20}$ film. Nevertheless, we are able to detect a response at distances up to $80 \mu \mathrm{m}$ from the CPS. From the data of the pulsed excitation the group velocities of the spin-wave packets are readily determined. The measured group velocities $v_{G}=\frac{\partial \omega}{\partial k}$ are highest for the DE geometry (up to $17 \mathrm{~km} / \mathrm{s}$ ), while they are smaller for geometries in between the DE and MSBV geometry and close to zero for the MSBV geometry. These values are in the same range as the ones found by Liu and co-workers, ${ }^{18}$ who found group velocities of $0.1 \mathrm{~km} / \mathrm{s}$ for the MSBV and $4.8 \mathrm{~km} / \mathrm{s}$ for a DE geometry (for a thinner film and at different bias fields).

We now turn to spin-wave interference experiments. For 

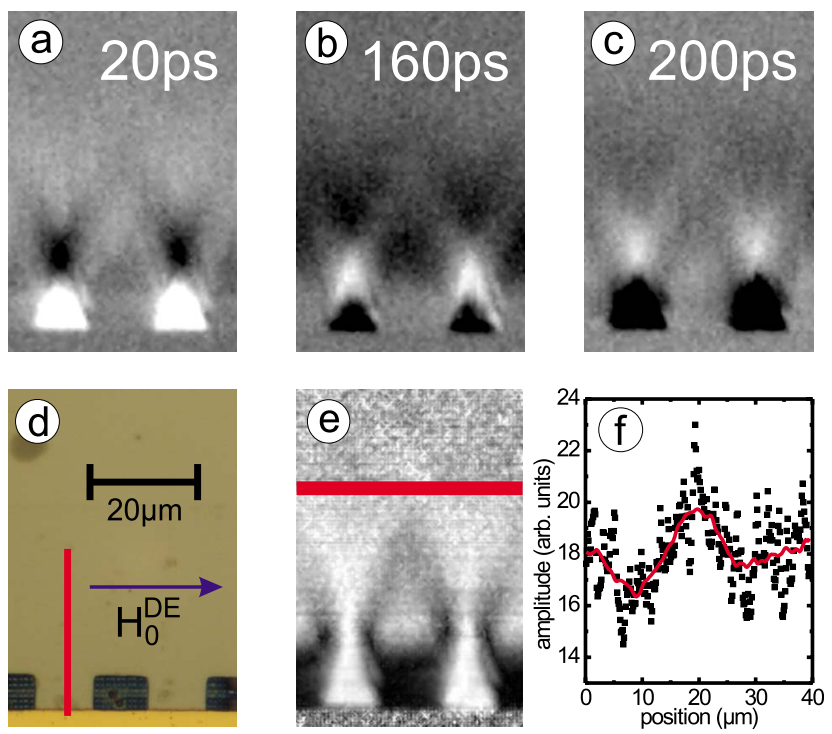

FIG. 4. (Color online) (a)-(c) Snapshots of the local magnetization configuration describing the decay of the signal) upon $\mathrm{cw}$ microwave excitation at $3.76 \mathrm{GHz}$ with a bias field of $17 \mathrm{mT}$ applied in the DE geometry. (d) Optical micrograph of the sample used for spin-wave interference experiments. Note the CPS (lower edge) and the two legs opening out into the continuous $\mathrm{Ni}_{80} \mathrm{Fe}_{20}$ film. The red line shows the position of the line scan shown in Fig. 5(a). (e) Distribution of the oscillation amplitude $m_{z}^{0}(\vec{x})$. The red line indicates the position of the line scan shown in (f). (f) Oscillation amplitude $m_{z}^{0}(\vec{x})$ along the line indicated in (e), individual data points (black squares), and adjacent averaged curve (red line). As expected for interference, the maximum of the amplitude is found at a position between the two legs (at $20 \mu \mathrm{m}$ ). The first minimum of the interference and the second interference maximum can be seen as well.

these experiments samples having two "legs" touching the CPS were prepared as shown in Fig. 4(b). The phase velocity of the microwave excitation inside the CPS is large enough to ensure an excitation of the two spin waves with equal phase. The external bias field used in our experiments is strong enough to saturate the magnetization in the sample, also inside the legs, and at the leg-film junction. This is supported by micromagnetic simulations (not shown). Spin waves are created in the legs close to the CPS and propagate towards the continuous $\mathrm{Ni}_{80} \mathrm{Fe}_{20}$ film. From the legs, spin waves propagate into the continuous $\mathrm{Ni}_{80} \mathrm{Fe}_{20}$ film where they can interfere. Thus, our experimental setup resembles a double-slit diffraction experiment for spin waves. The experimental results shown here have been obtained in the DE geometry. In Fig. 4(a) we show images of the dynamic magnetic response $m_{z}(\vec{x}, t)=m_{z}^{0}(\vec{x}) e^{-x / x_{0}} \sin (\vec{k} \vec{x}-\omega t+\varphi) \quad\left(x_{0}\right.$ describing the decay of the signal) at different phases with respect to the driving MW field. Obviously the two legs oscillate in phase. Spin waves first propagate inside the legs and then, as they reach the continuous film region, radiate into the continuous film. Here one observes a behavior that is different from the undisturbed propagation. Instead of a homogeneous wave front, fingerlike structures propagate from the legs. Snapshots of this are shown in Figs. 4(a)-4(c). Due to the anisotropic propagation (DE-like away from the CPS

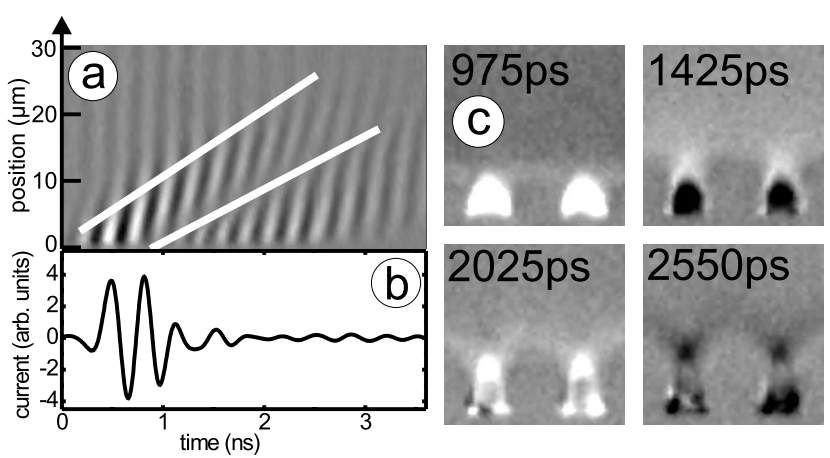

FIG. 5. Interference of spin-wave bursts. (a) Line scan through one leg of the interference structure at the position indicated in Fig. 4(d), (b) shape of the MWP excitation, and (c) snapshots of the propagating spin waves recorded at various delay times with respect to the onset of the MWP.

and MSBV-like parallel to the CPS), a distinct interference pattern can be observed that does not correspond to the interference pattern of circular wave fronts. Note the nodal lines (i.e., regions with zero oscillation amplitude) that can be observed in the snapshots and become even more pronounced when plotting the local distribution of the oscillation amplitude-i.e., $m_{z}^{0}(\vec{x})$ [see Fig. 4(e)]. Evaluating the oscillation amplitude along a line parallel to the CPS [see Fig. 4(f)], one finds a typical interference pattern with the main maximum, the first minimum, and the second maximum of interference.

Finally, we demonstrate MWP experiments on this sample. In Fig. 5(a) we show line scans as function of time, recorded at the position indicated in Fig. 4(d). One observes two spin-wave packets emitted from the legs; the first packet is directly excited by the microwave driving field, and the packet originates from the decay of standing waves inside the legs. ${ }^{22}$ Broadening of the spin-wave packets due to dispersion can be observed. In Fig. 5(b) the driving MW pulse is shown. Figure 5(c) shows images of the excitation recorded at delay times $\Delta t=975,1425,2025,2550 \mathrm{ps}$ after the MWP. Once again, a cross-shaped pattern is found due to the interference of the spin-wave bursts. In contrast to the interference with $\mathrm{cw}$ excitation, the spatial position of the pattern is not fixed in space but propagates away from the CPS due to the additional dispersion within the spin-wave packet.

In summary, the propagation of spin waves in thin ferromagnetic $\mathrm{Ni}_{80} \mathrm{Fe}_{20}$ films can be observed using TRMOKE microscopy. Phase velocities, dispersion relations, and group velocities are measured for different geometries of the inplane magnetic bias field. An analytical susceptibility calculation approach reproduces the experimentally determined dispersion relations. In patterned films, it is possible to directly observe the interference of spin waves with lateral and temporal resolution.

This work was supported by the DFG through priority program 1133 and Sonderforschungsbereich 689. K.P. acknowledges support by the Studienstiftung des Deutschen Volkes. We thank D. Weiss for permission to us use his clean room facilities. 
*georg.woltersdorf@physik.uni-r.de

${ }^{1}$ R. W. Damon, and J. R. Eshbach, J. Appl. Phys. 31, S104 (1960).

${ }^{2}$ P. C. Fletcher, and C. Kittel, Phys. Rev. 120, 2004 (1960).

${ }^{3}$ M. Sparks, Solid State Commun. 8, 731 (1970).

${ }^{4}$ J. F. Dillon, Jr., J. Appl. Phys. 31, 1605 (1960).

${ }^{5}$ G. Gubbiotti, G. Carlotti, T. Okuno, T. Shinjo, F. Nizzoli, and R. Zivieri, Phys. Rev. B 68, 184409 (2003).

${ }^{6}$ J. P. Park, P. Eames, D. M. Engebretson, J. Berezovsky, and P. A. Crowell, Phys. Rev. Lett. 89, 277201 (2002).

${ }^{7}$ C. Bayer, J. P. Park, H. Wang, M. Yan, C. E. Campbell, and P. A. Crowell, Phys. Rev. B 69, 134401 (2004).

${ }^{8}$ S. O. Demokritov, B. Hillebrands, and A. N. Slavin, Phys. Rep. 348, 441 (2001).

${ }^{9}$ M. Buess, T. P. J. Knowles, R. Hollinger, T. Haug, U. Krey, D. Weiss, D. Pescia, M. R. Scheinfein, and C. H. Back, Phys. Rev. B 71, 104415 (2005).

${ }^{10}$ C. H. Back, R. Allenspach, W. Weber, S. S. P. Parkin, D. Weller, E. L. Garwin, and H. C. Siegmann, Science 285, 864 (1999).

${ }^{11}$ Th. Gerrits, H. A. M. van den Berg, J. Hohlfeld, and L. Bär, and Th. Rasing, Nature (London) 418, 509 (2002).

${ }^{12}$ M. Wu, B. A. Kalinikos, P. Krivosik, and C. E. Patton, J. Appl.
Phys. 99, 013901 (2006).

${ }^{13}$ M. Covington, T. M. Crawford, and G. J. Parker, Phys. Rev. Lett. 89, 237202 (2002).

${ }^{14}$ M. Bailleuil, D. Olligs, C. Fermon, and S. O. Demokritov, Europhys. Lett. 56, 741 (2001).

${ }^{15}$ M. Bailleuil, D. Olligs, and C. Fermon, Appl. Phys. Lett. 83, 972 (2003).

${ }^{16}$ M. L. Schneider, Th. Gerrits, A. B. Kos, and T. J. Silva, Appl. Phys. Lett. 87, 072509 (2005).

${ }^{17}$ T. J. Silva, M. R. Pufall, and P. Kabos, J. Appl. Phys. 91, 1066 (2002).

${ }^{18}$ Z. Liu, F. Giesen, X. Zhu, R. Sydora, and M. Freeman, Phys. Rev. Lett. 98, 087201 (2007).

${ }^{19}$ I. Neudecker, K. Perzlmaier, F. Hoffmann, G. Woltersdorf, M. Buess, D. Weiss, and C. H. Back, Phys. Rev. B 73, 134426 (2006).

${ }^{20}$ G. Woltersdorf and C. H. Back, Phys. Rev. Lett. 99, 227207 (2007).

${ }^{21}$ K. J. Harte, J. Appl. Phys. 39, 1503 (1968).

${ }^{22}$ V. E. Demidov, B. Hillebrands, S. O. Demokritov, M. Laufenberg, and P. P. Freitas, J. Appl. Phys. 97, 10A717 (2005). 